\title{
Metabolic Labeling of Yeast Proteins
}

Bio-protocol Editor*

Bio-protocol LLC, Sunnyvale, CA 94087, USA

*For correspondence: team@ed.bio-protocol.org

[Abstract] Proteins of Saccharomyces cerevisiae can be metabolically labeled with (35) methionine. After labeling, a protocol is described for the mechanical disruption of yeast cells or conversion to spheroplasts, with subsequent lysis before immunoprecipitation of the proteins.

\section{Materials and Regents}

1. TRAN-35'-label (MP Biomedicals)

2. Methionine

3. FLAG peptides

4. Immunoprecipitation buffer

5. Digitonin (EMD Chemicals)

6. $1 \times$ SDS

7. Medium minus methionine (SD-Met)

\section{Equipment}

1. Bench-top centrifuges

2. Charcoal filter paper

3. $50 \mathrm{ml}$ conical tube

4. $125 \mathrm{ml}$ Erlenmeyer flask with vented cap (Nalgene)

5. O-ring cap tube

6. Geiger counter

7. Labeled radioactive beaker

8. Kimwipes

9. $1.5 \mathrm{ml}$ Eppendorf tubes 


\section{Procedure}

1. The evening before labeling, set up several $25 \mathrm{ml}$ cultures, inoculated at different densities, so that first thing the next morning, at least one of the cultures will be at an OD of $\sim 0.5$.

2. Labeling requires a $25 \mathrm{ml}$ culture of yeast at an $\mathrm{OD}$ of between 0.4 and 0.6 . When the $\mathrm{OD}$ of the culture is $\sim 0.4$ to 0.6 , transfer the culture to a $50 \mathrm{ml}$ conical tube. Centrifuge at $2,000 \mathrm{rpm}$ for $5 \mathrm{~min}$ at room temperature (RT).

3. Pour off supernatant. Resuspend the yeast pellet in $25 \mathrm{ml}$ of warm medium minus methionine (SD-Met). Return cells to flask and grow for $1 \mathrm{~h}$.

If temperature-sensitive yeast is used, shift to the restrictive temperature during the last 30 min of the starvation period.

4. Read the OD of the culture. Transfer the culture to a $50 \mathrm{ml}$ conical tube. Centrifuge at 2,000 rpm for 5 min at RT.

5. Pour off supernatant. Resuspend the pellet in $15 \mathrm{ml}$ warm medium minus methionine (SD-Met) to give a final OD of 1 (total OD of 15). Transfer it to Nalgene $125 \mathrm{ml}$ Erlenmeyer flask with vented cap.

6. Add $100 \mathrm{Ci}$ of TRAN35S-label (a mixture of $\left[{ }^{35} \mathrm{~S}\right.$ ] methionine and $\left[{ }^{35} \mathrm{~S}\right]$ cysteine) per $\mathrm{ml}$, e.g. $1.5 \mathrm{mCi}$ for $15 \mathrm{ml}$ culture). Incubate at $37^{\circ} \mathrm{C}$ for $2 \mathrm{~h}$. The total $\mathrm{OD}$ will probably reach 25 OD.

Thaw [35S] in the hood. Use pipettes with filters. Wrap the flask with charcoal filter paper.

7. Pour the labeled culture into a $50 \mathrm{ml}$ conical tube. Use Kimwipe to wipe off the last drop from the Nalgene flask. Spin down yeast cells in Centrifuge 5810R at 3,000 rpm for $3 \mathrm{~min}$. Wear $3 \mathrm{M}$ mask while unwrapping the charcoal filter paper, which is contaminated with S35 labeled volatile acids.

8. Resuspend cells in $10 \mathrm{ml}$ of $\mathrm{H}_{2} \mathrm{O}$. Wash twice. Proceed to small scale native affinity purifications of solubilized membrane proteins protocol.

9. Prepare $10 \mathrm{ml}$ of $\mathrm{H}_{2} \mathrm{O}$ in $15 \mathrm{ml}$ conical tubes. Pour water into tubes containing labeled cells, close cap and swirl the tube to resuspend cell pellet.

10. For the last wash, leave $\sim 1 \mathrm{ml}$ supernatant. Resuspend the pellet and transfer the cell suspension into an o-ring cap tube using a $5 \mathrm{ml}$ serological pipette.

Avoid using Pipetman, which can be contaminated during the procedure. Serological pipettes can be disposed to radioactive solid waste. 


\section{Notes}

Important notice for performing small-scale purification of solubilized membrane proteins from S35 labeled yeast extracts.

1. General advice: Use o-ring cap tubes instead of $1.5 \mathrm{ml}$ Eppendorf tubes; Always use barrier pipette tips instead of regular ones; tips and serological pipettes that touch radioactive material should be disposed in radioactive waste.

2. Add one scoop of glass beads into an empty o-ring cap tube. Then transfer S35 labeled cell suspension $(\sim 150 \mu \mathrm{l})$ into the tube containing glass beads.

3. Dilute the supernatant from $100,000 \times g$ spin. Dilute $800 \mu \mathrm{l}$ supernatant to $4 \mathrm{ml}$ in total.

4. After incubation with ANTI-FLAG M2 affinity gel, spin down the resin. Use serologicalpipette to transfer $3 \mathrm{ml}$ supernatant to radioactive liquid waste. Transfer $1 \mathrm{ml}$ resin containing solution into a fresh o-ring cap tube. No need to save input or unbound, which is too radioactive on the membrane.

5. Wash the resin 4 times with $1 \mathrm{ml}$ immunoprecipitation buffer with $0.1 \%$ digitonin as usual. You can use a labeled radioactive beaker for liquid waste temporarily, and then dump all the waste to designated radioactive liquid waste.

6. After elution with FLAG peptides, collect bound proteins. Then add 1x SDS dye to the resin and boil it. The resin is still quite radioactive, handle with respect.

Important notice for running SDS-PAGE and Western Blot transfer with radioactive material.

1. Load the gel with radioactive samples in a designated radioactive area.

2. Assemble cassette for transfer in the designated area. After transfer, use Geiger counter to check membrane, gel, filter paper, etc. Usually, the membrane is radioactive, while the rest is not.

3. Place the protein side of the membrane onto Saran Wrap, which will not block radioactivity. Place aluminum foil on the back side of the membrane to block radiation. Tape this onto a film cassette and place a film on the protein side of the membrane.

\section{Acknowledgments}

This protocol has been modified and adapted in the Espenshade Lab, Johns Hopkins School of Medicine. Funding to support different projects that have used this protocol has come from $\mathrm{NIH}$ - National Heart, Lung, and Blood Institute, National Institute of Allergy and Infectious Diseases, the Pancreatic Cancer Action Network, and the American Heart Association. 


\section{References}

1. Sacher, M., Jiang, Y., Barrowman, J., Scarpa, A., Burston, J., Zhang, L., Schieltz, D., Yates, J. R., 3rd, Abeliovich, H. and Ferro-Novick, S. (1998). TRAPP, a highly conserved novel complex on the cis-Golgi that mediates vesicle docking and fusion. EMBO J 17(9): 2494-2503. 